\title{
Extracellular matrix-associated proteins form an integral and dynamic system during Pseudomonas aeruginosa biofilm development
}

\author{
Weipeng Zhang ${ }^{1+}$, Jin Sun ${ }^{2 t}$, Wei Ding ${ }^{3}$, Jinshui Lin ${ }^{3}$, Renmao Tian ${ }^{1}$, Liang Lu ${ }^{1}$, \\ Xiaofen Liu ${ }^{1}$, Xihui Shen ${ }^{3}$ and Pei-Yuan Qian ${ }^{1 *}$ \\ ${ }^{1}$ Division of Life Science, The Hong Kong University of Science and Technology, Hong Kong, China, ${ }^{2}$ Department of Biology, \\ Hong Kong Baptist University, Hong Kong, China, ${ }^{3}$ State Key Laboratory of Crop Stress Biology for Arid Areas and College \\ of Life Sciences, Northwest A \& F University, Yangling, China
}

OPEN ACCESS

Edited by:

Jiri Stulik,

University of Defence, Czech Republic

Reviewed by:

Odile Tresse,

French National Institute for Agricultural Research/Nantes-Atlantic National College of Veterinary Medicine, Food Science and

Engineering, France Jay Nadeau,

McGill University, Canada

${ }^{*}$ Correspondence:

Pei-Yuan Qian,

Division of Life Science, The Hong Kong University of Science and

Technology, Clear Water Bay,

Hong Kong, China boqianpy@ust.hk

${ }^{t}$ These authors have contributed equally to this work.

Received: 16 December 2014 Accepted: 27 April 2015 Published: 13 May 2015

Citation:

Zhang W, Sun J, Ding W, Lin J, Tian R, Lu L, Liu X, Shen X and Qian P-Y (2015) Extracellular matrix-associated proteins form an integral and dynamic system during Pseudomonas aeruginosa biofilm development.

Front. Cell. Infect. Microbiol. 5:40 doi: 10.3389/fcimb.2015.00040
Though the essential role of extracellular matrix in biofilm development has been extensively documented, the function of matrix-associated proteins is elusive. Determining the dynamics of matrix-associated proteins would be a useful way to reveal their functions in biofilm development. Therefore, we applied iTRAQ-based quantitative proteomics to evaluate matrix-associated proteins isolated from different phases of Pseudomonas aeruginosa ATCC27853 biofilms. Among the identified 389 proteins, 54 changed their abundance significantly. The increased abundance of stress resistance and nutrient metabolism-related proteins over the period of biofilm development was consistent with the hypothesis that biofilm matrix forms micro-environments in which cells are optimally organized to resist stress and use available nutrients. Secreted proteins, including novel putative effectors of the type III secretion system were identified, suggesting that the dynamics of pathogenesis-related proteins in the matrix are associated with biofilm development. Interestingly, there was a good correlation between the abundance changes of matrix-associated proteins and their expression. Further analysis revealed complex interactions among these modulated proteins, and the mutation of selected proteins attenuated biofilm development. Collectively, this work presents the first dynamic picture of matrix-associated proteins during biofilm development, and provides evidences that the matrix-associated proteins may form an integral and well regulated system that contributes to stress resistance, nutrient acquisition, pathogenesis and the stability of the biofilm.

Keywords: biofilm, matrix-associated proteins, iTRAQ, type three secretion system, Pseudomonas aeruginosa ATCC27853

\section{Introduction}

Infections caused by bacterial biofilms, which are composed of microorganisms that attach to a surface, have emerged as a major public health concern. Biofilm development occurs in sequential processes in general: attachment (phase I), microcolony formation (phase II), maturation I 
(phase III), maturation II (phase IV), dispersal (phase V) (O'Toole et al., 2000; Waite et al., 2005). In a biofilm, cells are embedded in the extracellular polymeric substance (EPS), also known as the extracellular matrix. The extracellular matrix consists (hereafter referred as matrix) of nucleic acids, polysaccharides, lipids and proteins. Several studies (HallStoodley et al., 2004; Flemming and Wingender, 2010; Colvin et al., 2011; Lewenza, 2013) have shown that polysaccharides and DNA in the matrix play important roles in biofilm development. For example, polysaccharides provide mechanical stability, mediate bacterial adhesion to surfaces and form a cohesive, three-dimensional network that connects and immobilizes biofilm cells. Compared to polysaccharides, information about matrix-associated proteins is limited. Matrix-associated proteins have been identified from some microorganisms, such as Haemophilus influenzae biofilm that contains over 200 proteins involved in cell motility and secretion (Gallaher et al., 2006). In a recent study, the role of extracellular matrix binding protein in Staphylococcus aureus biofilm formation was reviewed (Speziale et al., 2014). However, the dynamics of matrix-associated proteins during biofilm development have not been systematically studied, and their roles in biofilm development remain elusive.

Nutrient acquisition, stress resistance and pathogenesis are important processes associated with biofilm development. Biofilm development is largely affected by nutrients that are available in the environment. For example, specific L-amino acids are required for the formation of a tight microcolony as well as various cysticfibrosis-specific phenotypes of Pseudonomas aeruginosa PAO1 (Sriramulu et al., 2005). Nutrients such as sucrose, phosphate and calcium enhance biofilm formation of Sinorhizobium meliloti as their concentrations increase (Rinaudi et al., 2006). In addition, biofilm development is associated with enhanced resistance to environmental stresses such as oxidative stress, antibiotics and host immune response (Mah and O'Toole, 2001; Arciola et al., 2005; Zhang et al., 2013a). The mechanisms underlying these types of resistance have been attributed to the expression of biofilm-specific genes and phenotypic changes (Mah and O'Toole, 2001; Arciola et al., 2005; Zhang et al., 2013a). Moreover, biofilm development has also been associated with a range of infections, whereas polysaccharide components of the biofilm matrix play roles in pathogenesis and facilitate biofilm development in the host (Goller and Seed, 2010).

$P$. aeruginosa is a model organism for biofilm research in the laboratory (Stewart et al., 1993). In the present study, we investigated the dynamics of matrix-associated proteins in biofilm development by $P$. aeruginosa ATCC27853. P. aeruginosa ATCC27853 is a clinical strain that is frequently used in antimicrobial susceptibility testing (Fass and Barnishan, 1979), and its draft genome was sequenced in 2012 (Fang et al., 2012). The genetic and molecular bases underlying biofilm development by this bacterial strain remains largely unknown. Using iTRAQbased proteomic analysis (Wiese et al., 2007) to quantify matrixassociated proteins isolated from $P$. aeruginosa ATCC27853 biofilms in phases I-IV, we discovered significant changes in protein related to nutrient metabolism, stress resistance and pathogenesis. Subsequently, we investigated gene expression, protein-protein interactions and the influence of gene mutations on biofilm development.

\section{Materials and Methods}

\section{Bacterial Strains, Culture Media, and Biofilm Development}

The bacterial strains and plasmids used in this study are listed in Supplementary Table 1. Escherichia coli JM109 was used for the cloning experiments, and $E$. coli 17-1 $\lambda$ pir was used for the conjugation experiments. $P$. aeruginosa ATCC27853 was obtained from China General Microbiological Culture Collection (CGMCC). P. aeruginosa ATCC27853 was grown at $37^{\circ} \mathrm{C}$ in $\mathrm{M} 9$ broth or on $\mathrm{M} 9$ agar plates containing $50 \mu \mathrm{g}$ $\mathrm{mL}^{-1}$ kanamycin. Biofilms were developed using a static model according to the method described by Waite et al. (2005) with modifications. Briefly, nitrocellulose filters (diameter, $47 \mathrm{~mm}$; Millipore, Bedford, MA, USA) placed on M9 agar were incubated with $10^{5} \mathrm{CFU}$ of bacterial culture at $37^{\circ} \mathrm{C}$ before monitored at different time points.

\section{Microscopic Observation and Characterization}

Biofilms were stained with fluorescein isothiocyanate (FITC) (Sigma, Poole, United Kingdom). Microscopic observations were performed using a Zeiss LSM 510 CLSM (Carl Zeiss, Jena, Germany) equipped with detectors for FITC. Images were obtained using a $63 \times / 1.4$ objective, and signals were recorded in the green channel (excitation $488 \mathrm{~nm}$, emission 522/32 nm). The stacked CLSM images were then analyzed for microbial cell density, biovolume, mean thickness, maximum thickness and coverage using the image quantification tool PHLIP34.

\section{Exopolysaccharide Quantification}

The concentration of exopolysaccharides in the biofilms was measured according to previously reported procedures (Myszka and Czaczyk, 2009). Biofilm samples were thawed on ice and centrifuged at 15,000 $\mathrm{g}$ for $20 \mathrm{~min}$. The pellets were re-suspended in $\sim 3 \mathrm{~mL}$ of a cold sulfuric acid solution ( $0.2 \mathrm{M}$ sulfuric acid, $\mathrm{pH}$ 1.1), and the biofilm matrix was broken using a glass hand homogenizer tube and pestle before centrifugation at 15,000 $\mathrm{g}$ for $20 \mathrm{~min}$. The resulting supernatant was collected and suspended in water, followed by the addition of $1.4 \mathrm{~mL}$ of $77 \%(\mathrm{v} / \mathrm{v}) \mathrm{H}_{2} \mathrm{SO}_{4}$. Subsequently, the samples were mixed with $200 \mu \mathrm{L}$ of $1 \%(\mathrm{w} / \mathrm{v})$ cold tryptophan. Finally, they were heated at $37^{\circ} \mathrm{C}$ for $20 \mathrm{~min}$, and the colored product was evaluated at $\mathrm{OD}_{500}$. Calibration curves were prepared using dextran solution.

\section{Genome Annotation}

The draft genome sequence of $P$. aeruginosa strain ATCC27853 was downloaded from the NCBI bacterial genome database. Putative reading frames were identified using Glimmer v.3.0 (Delcher et al., 2007). For the annotation, BLAST searches were performed against clusters of orthologous group (COG, Version 9.05) (Tatusov et al., 2001), KEGG (Kanehisa and Goto, 2000), CAZy (Cantarel et al., 2009) and P. aeruginosa strain PAO1 according to procedures reported in our previous work (Zhang et al., 2013a). We used prokaryote databases on local servers and 
used an $E$-value cut-off of $<10^{-5}$. As a considerable number of hypothetical proteins were identified, the annotation of selected proteins was confirmed by a manual BLAST search against the NCBI non-redundant database.

\section{Extraction of Matrix-associated Proteins}

The matrix-associated proteins were isolated from the biofilms of $12,24,48$, and $96 \mathrm{~h}$ respectively. Matrix-associated protein preparations were obtained by referring to classical methods described previously (Zhang et al., 1999; Liu and Fang, 2002). The "regular centrifuge" method was used with modifications. Briefly, the biofilms were scraped from the medium and re-suspended in cold $0.9 \% \mathrm{NaCl}$ prior to incubation at $4{ }^{\circ} \mathrm{C}$ for $2 \mathrm{~h}$. After centrifugation for $30 \mathrm{~min}$ at $4000 \mathrm{~g}$ at $4^{\circ} \mathrm{C}$, the supernatant was filtered through a $0.22-\mu \mathrm{m}$ pore-sized filter to remove bacterial cell contamination. The supernatant was then precipitated by the addition of 3 times the volume of acetone. After storage at $-20^{\circ} \mathrm{C}$ overnight, matrix-associated proteins were pelleted by centrifugation for $30 \mathrm{~min}$ at $4000 \mathrm{~g}$ at $4^{\circ} \mathrm{C}$. The protein pellet was re-suspended in a buffer containing $8 \mathrm{M}$ urea and $40 \mathrm{mM}$ HEPES ( $\mathrm{pH} 7.5)$.

\section{iTRAQ Protein Quantification Analysis}

Protein labeling and strong cation exchange fractionation were performed as described in our previous study (Han et al., 2013). Protein collected from each sample was purified using the ReadyPrep 2-D Cleanup Kit (Bio-Rad, Richmond, CA) and quantified using the RC-DC kit (Bio-Rad, Richmond, CA). Subsequently, $200 \mu \mathrm{g}$ of protein from each sample was reduced, alkylated and digested with trypsin. The desalted peptides were labeled with four different iTRAQ compounds: 114, 115, 116, and 117 for matrix-associated proteins from biofilms of 12, 24,48 , and $96 \mathrm{~h}$, respectively. After labeling, the samples were mixed, dried and fractionated using a strong cation exchange (SCX) column. The peptides were subjected to high-pressure liquid chromatography (HPLC) and the quadrupole time-offlight (QSTAR XL) MS system.

Protein identification and quantification also were performed as previously described (Han et al., 2013). Briefly, raw MS/MS data were converted into non-deisotoped and deisotoped $p k l$ files using Proteinlynx Global SERVER 2.2.5 (Waters Corp, Milford, MA, USA). The reporter mass (114-118 Da) was extracted from the non-deisotoped file, and the deisotoped files were replaced with the same mass. The combined pkl files were submitted to Mascot version 2.3.0 (Matrix Science, Ltd., London, UK) to search against translated forward and reverse $P$. aeruginosa ATCC27853 protein sequences. The minmium number of peptides for protein is 2 . We removed peptide scores that is less than $95 \%$ confidence identification level. We also performed a "target-decoy" database search, and the false discovery rate (FDR) in this analysis was controlled as less than $1 \%$. Because $99 \%$ of the identified proteins varied 1.4-fold between the three experimental replicates (technical replicates that started from peptide labeling), the cut-off for the identification of significantly changed proteins was set at 1.4, corresponding to a $99 \%$ confidence level in the quantification analysis.

\section{Quantitative PCR (qPCR)}

RNA was extracted from 40 Petri dishes containing biofilms using the ALLPrep DNA/RNA Mini Kit (Qiagen, Hilden, Germany). To remove potential DNA contamination, the TURBO DNAfree TM Kit was applied (Applied Biosystems, Foster City, CA). Subsequently, RNA was reverse transcribed into first-strand cDNA using the SuperScript III First-Stand Synthesis SuperMix Kit (Invitrogen, Carlsbad, CA) and random hexamers. The qPCR reactions were conducted using the Kapa SYBR Fast qPCR Kit (Kapa Biosystems, Woburn, MA) on a Mx3000P qPCR System (Agilent Technologies, Palo Alto, CA). Each $20-\mu \mathrm{l}$ qPCR reaction contained $10 \mu \mathrm{l}$ of $2 \times$ Master Mix, $1 \mathrm{pmol} / \mu \mathrm{l}$ of forward and reverse primers, and $0.5 \mu \mathrm{l}$ of either standard or environmental sample. The cycling parameters were $5 \mathrm{~min}$ at $95^{\circ} \mathrm{C}$, followed by 40 cycles of $15 \mathrm{~s}$ at $95^{\circ} \mathrm{C}, 15 \mathrm{~s}$ at $50^{\circ} \mathrm{C}$ and $15 \mathrm{~s}$ at $60^{\circ} \mathrm{C}$. The $16 \mathrm{~S}$ rRNA gene was used as an internal standard in $\mathrm{qPCR}$, and the fold change of one target gene from different biofilm stages was calculated by normalizing its $\mathrm{Ct}$ values to the $\mathrm{Ct}$ values of the $16 \mathrm{~S}$ rRNA gene. Three experimental replicates (technical replicates that started from cDNA synthesis) were performed. The primers used in this study are listed in Supplementary Table 2.

\section{Construction of Mutant Strains}

Mutant strain construction was conducted following the procedure described in our previous study (Zhang et al., 2013b).

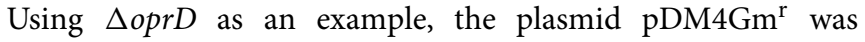
constructed by inserting SaI- and BglII-digested $\mathrm{Gm}^{\mathrm{r}}$ fragments (amplified using $\mathrm{Gm}^{\mathrm{r}}$-SalI-F/ $\mathrm{Gm}^{\mathrm{r}}$-XhoI-R and pZL184 as a template) into the SalI site of pDM4. The resulting plasmid pDM4Gm ${ }^{\mathrm{r}}-\Delta o p r D$ was used to construct the oprD deletion mutant of $P$. aeruginosa ATCC27853. Upstream and downstream PCR products of oprD were amplified using primer pairs $\Delta o p r D u p-S a l I-F / \Delta o p r D u p-R$ and $\Delta o p r D d o w n-F / \Delta o p r D d o w n-$ EcoRI-R, respectively. The upstream and downstream products were then linked together by overlap PCR and inserted into the SalI/EcoRI sites of pDM4Gmr to generate pDM4Gm ${ }^{\mathrm{r}}-\Delta o p r D$. Equal amounts of late logarithmic-phase cultures of E. coli S17$1 \lambda$ pir grown at $30^{\circ} \mathrm{C}$ with shaking and of strain ATCC27853 grown at $37^{\circ} \mathrm{C}$ without shaking were mixed and grown on $\mathrm{LB}$ agar. Cells from the agar plates were suspended in LB medium, and a strain with a single crossover event was selected on minimal medium containing $100 \mu \mathrm{g} \mathrm{mL}^{-1} \mathrm{Gm}$. Strains with double crossovers were counter-selected using LB agar plus $10 \%$ sucrose.

\section{Results}

\section{Developmental Processes of the $P$. aeruginosa ATCC27853 Biofilm}

As shown in Figure 1, after $12 \mathrm{~h}$ of growth on M9 medium, $P$. aeruginosa ATCC27853 cells remained separate but became aggregated and formed flat microcolonies with irregular shapes at $24 \mathrm{~h}$. A large number of swimming bacteria were present in the first two phases of biofilm development. At $48 \mathrm{~h}$, the cells became embedded in the matrix and complex three-dimensional structure began to form. At $96 \mathrm{~h}$, the bacteria eventually formed multilayers, resulting in complex structures with a maximum 


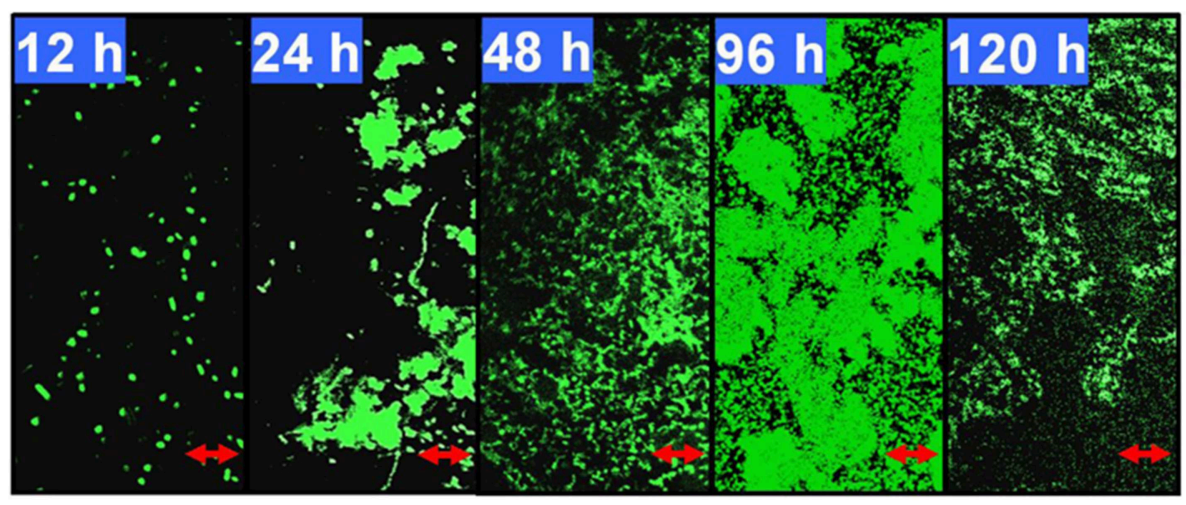

FIGURE 1 | Developmental processes of the Pseudomonas aeruginosa ATCC27853 biofilm. Biofilms were developed using a static model according to the method described by Waite et al. (2005). The 12, 24, 48,96 , and 120 -h-old biofilms were monitored by confocal laser scanning microscopy (CLSM). These five time points correspond to phases I-V, respectively, in this biofilm model. The scale bars are $10 \mu \mathrm{m}$. The coverage, thickness and biomass of the biofilms at different phases are shown in Supplementary Table 3.

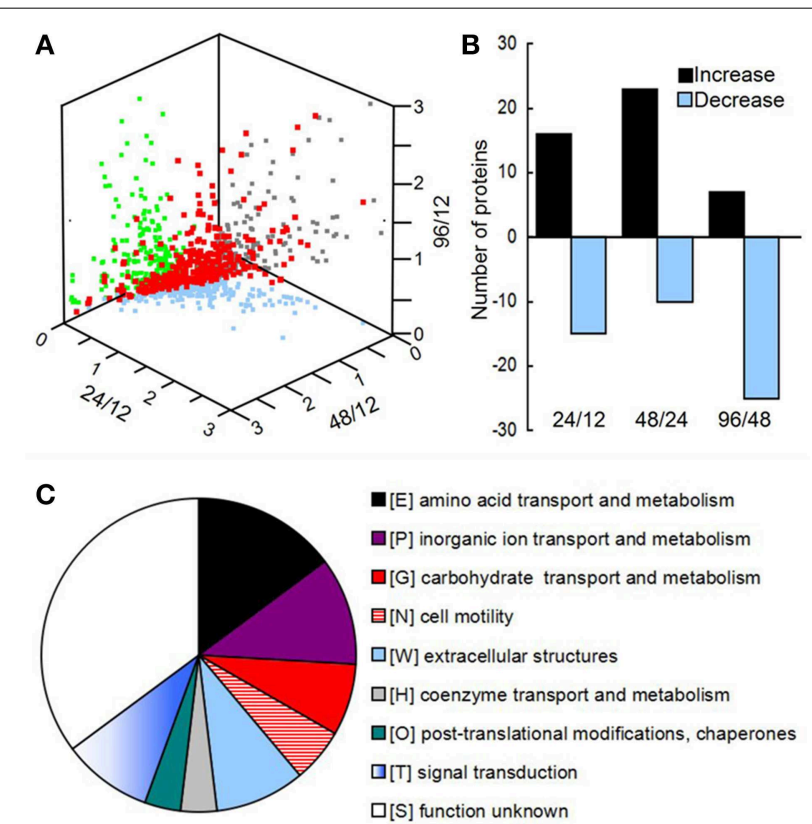

FIGURE 2 | (A) Three-dimensional diagram representing the distribution of all identified proteins using the abundance ratios between different phases as the $\mathrm{X}$-, Y-, and Z-axes, respectively. In total, 389 proteins were quantified, of which 54 showed significant changes among the different phases. (B) The numbers of proteins with different abundances from 12 to $24 \mathrm{~h}, 24$ to $48 \mathrm{~h}$, and 48 to $96 \mathrm{~h}$. (C) Clusters of orthologous groups (COGs) of proteins with different abundances in the matrix during biofilm development.

thickness. Detachment was observed for biofilms that developed for $120 \mathrm{~h}$. The coverage, thickness and biomass also were measured to confirm the phase classification (Supplementary Table 3). The biofilm reached its maximum biomass and coverage at $96 \mathrm{~h}$. Therefore, in this model, growth for 12, 24, 48, and $96 \mathrm{~h}$ coincided with phases I-IV of biofilm development by $P$. aeruginosa ATCC27853, respectively.

\section{Comparative Quantification of Matrix-associated Proteomes from Different Phases of Biofilm Development}

Using OFFGEL fractionation of the pooled iTRAQlabeled peptides and LC-MS/MS analysis, we identified 389 proteins (Supplementary File 1) from 5143 unique sequences. Comparison of the biofilm proteomes from different developmental phases revealed that 54 proteins had significantly different ( $\geqq 1.4$-fold) abundance (Figure 2A and Supplementary Table 4). From phase 12 to $24 \mathrm{~h}$ and 24 to $48 \mathrm{~h}$, more proteins had an increased abundance than those with a decreased abundance; but from phase 48 to $96 \mathrm{~h}$, most of the modulated proteins showed a decreased abundance (Figure 2B). Key protein structural components of the biofilm matrix were identified as CdrA and CupB5, which showed the highest abundance in phase I; this result was consistent with their roles in promoting biofilm formation and auto-aggregation (Borlee et al., 2010; Giraud et al., 2010). Moreover, the FlgM protein, which is secreted into the matrix in response to flagellar hook-basal body secretion (Guo et al., 2014), decreased by four-fold during biofilm development, which was consistent with the notion that motility is inhibited by biofilm development. The top 20 highly abundant proteins are listed in Supplementary Table 5, and most of them were secreted proteins such as lipoproteins and outer membrane proteins such as OmpA. All these results suggested that the method applied in this study to extract matrix-associated proteins was successful.

All of the significantly changed proteins were grouped into several COG categories (Figure 2C and Supplementary Table 4). Proteins related to nutrient metabolism and acquisition changed in abundance in the matrix during biofilm development (Figure 3A and Supplementary Table 4). The category with the largest number of proteins was amino acid transport and metabolism, which consisted of Aminopeptidase, OmpA, IlvC, IlvE, OprD, and OprE, among others. Most of these proteins showed an increased abundance from phases I-IV. For example, the abundance of porin OmpA increased by four-fold during biofilm development. In addition to proteins classified under the 


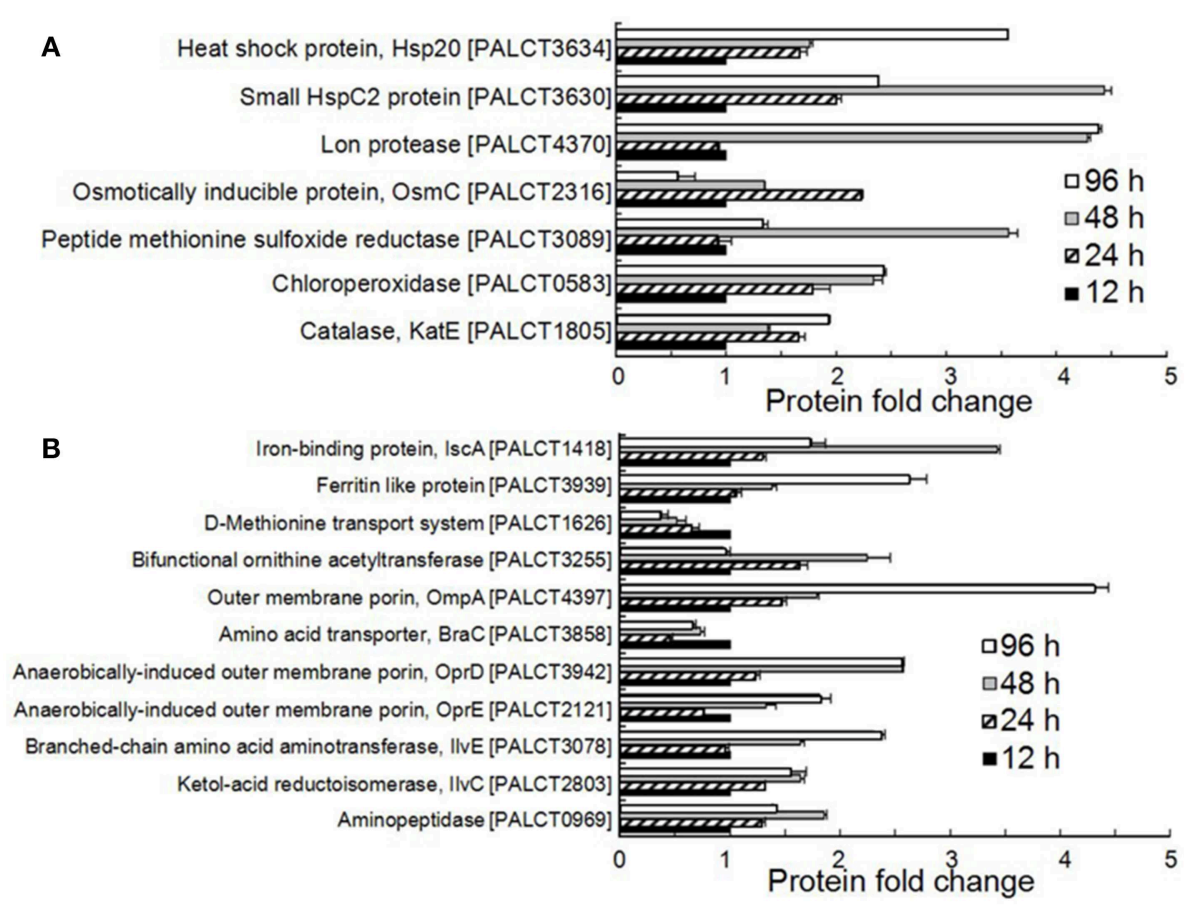

FIGURE 3 | Significantly changed proteins associated with stress resistance (A) and nutrient metabolism and acquisition (B) in the biofilm matrix. Average values from three experimental replicates are shown.

category of amino acid transport and metabolism, other proteins related to nutrient acquisition also displayed significant changes, such as the iron-binding protein IscA.

Many proteins related to the stress response were identified and were significantly changed in abundance in the matrix (Figure 3B and Supplementary Table 4). These include enzymes responsible for resistance to mainly oxidative stress and heat shock stress. For example, the abundance of catalase (CatE), chloroperoxidase and methionine sulfoxide reductase increased during biofilm development. Catalases are $\mathrm{H}_{2} \mathrm{O}_{2}$ dismutases, chloroperoxidases are $\mathrm{H}_{2} \mathrm{O}_{2}$ reductant oxidoreductases (Hewson and Hage, 2012), and methionine sulfoxide reductase is also required by bacteria to cope with oxidative stress (Sansom et al., 2013). In addition, the abundance of two heat shock proteins, the small HspC2 protein and Hsp20, also increased during biofilm development.

Some of the changed proteins were secreted proteins related to pathogenesis, such as chitin-binding protein $\mathrm{CbpD}$ and elastase LasB, which increased in abundance in the late phases compared with the early phases of biofilm development. Efforts were taken to identify the putative functions of the hypothetical proteins by searching against known databases including COG, KEGG (version 67.0), CAZy and Effective (Jehl et al., 2001). As a result, three hypothetical proteins hits were found in the Effective database. Based on the detectable secretion signal for the type three secretion pathways, three proteins (PALCT0513, PALCT3445, and PALCT3741) were identified as putative effectors of the type III secretion system (TTSS). To obtain more supporting evidence, the three-dimensional structures of the three putative effectors were predicted using QUARK protein structure prediction software (Xu and Zhang, 2012). The three putative TTSS effectors displayed similar structures to known TTSS effectors (Figure 4A), which contain a signal peptide at the $\mathrm{N}$-terminus and several helices (Lilic et al., 2006). Conserved beta motif-like sequences for chaperone binding also were identified based on sequence alignment with a previous study (Figure 4B) (Lilic et al., 2006).

\section{Expression Profile of Matrix-associated Proteins}

As mentioned above, proteins related to nutrient metabolism, stress response and pathogenesis showed a significant change in abundance in the matrix during biofilm development. To determine whether these dynamics were consistent with the expression of the proteins, RNA was extracted from biofilms of $12,24,48$, and $96 \mathrm{~h}$, and the expression levels of 20 selected genes were compared using qPCR (see results in Figure 5). Among the examined 20 genes, the expression levels of five genes, including las $B$, phoP, ompA, and katE, were not consistent with the protein expression levels. For the remaining 15 genes, there was a good correlation of the results in the proteomic analysis and the RT-PCR assay (Supplementary Figure 1). These findings suggested that the abundance of most matrix-associated proteins was affected by their expression levels and that the dynamics of matrix-associated proteins could be regulated by the bacterial cells.

\section{Protein-protein Interaction Networks and Gene Mutations}

Because several changed proteins in the matrix could be categorized under the same function (such as oxidative stress 


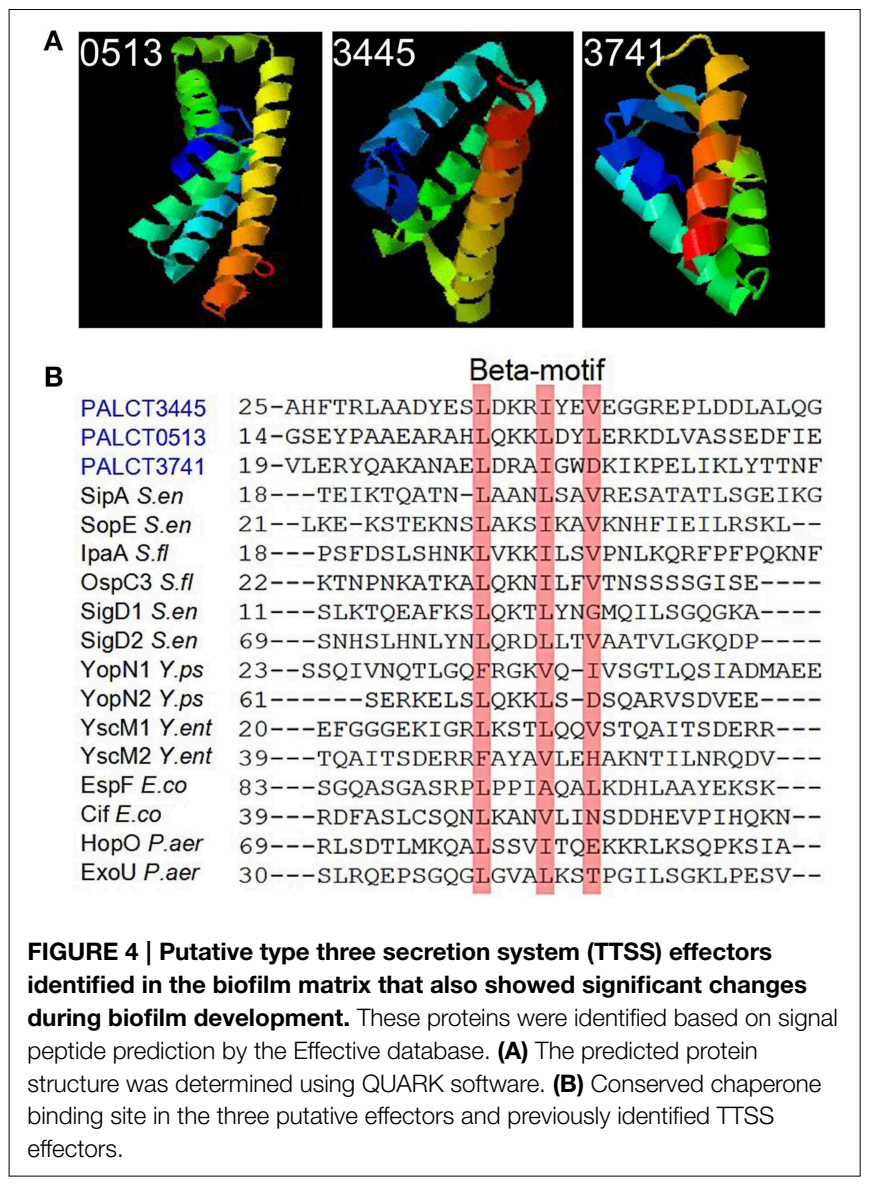

resistance), we speculated that these proteins interacted. Using the software STRING (Von Mering et al., 2003), an interaction network was built and shown in Figure 6; and many of these proteins were closely related to one another. Specifically, at the center of the network, OmpA interacted with OprE, OmpH, and Lon protease. The central network position of the OprD, OmpA, and CbpD proteins suggested that they exert important roles in P. aeruginosa ATCC27853 biofilms. To confirm this assumption, mutant strains of the three genes were obtained, and were compared with the wild-type stain. Indeed, the mutant strains CbpD and OmpA showed a decreased biofilm thickness, coverage and exopolysaccharide concentration at the maturation phase the OprD mutation affected the maximum thickness of the biofilm (Table 1).

\section{Discussion}

Based on study of polysaccharides in the matrix, it has been hypothesized that the biofilm matrix is a dynamic environment in which microbial cells are optimally organized to use the available nutrients and resist stress (Hall-Stoodley et al., 2004; Flemming and Wingender, 2010). Here, we provide evidence to support this hypothesis based on the study of the dynamics of matrix-associated proteins. The results in the present study also suggest that the matrixassociated proteins, functioning as an integral system, contribute to pathogenesis and to the stability of the biofilm. Therefore, determination of the dynamics of matrix-associated proteins is a useful way to reveal their roles in biofilm development.

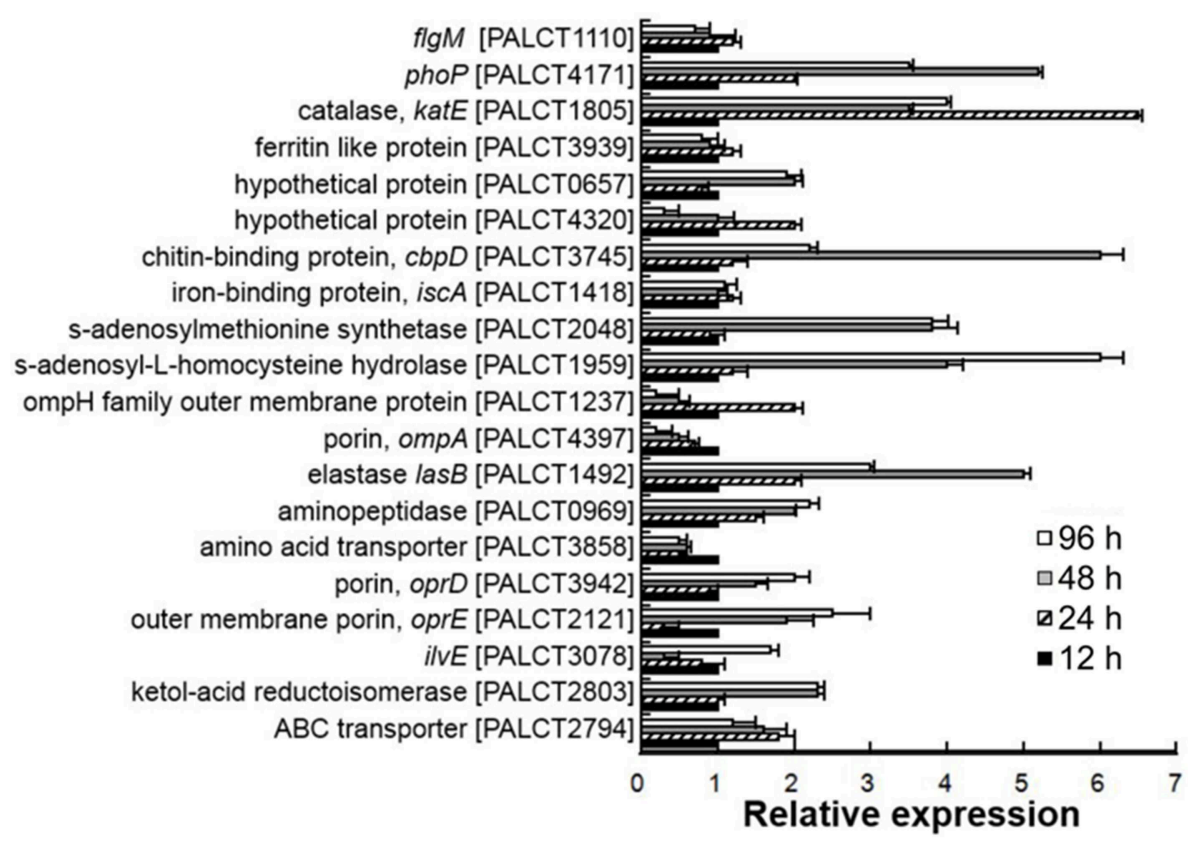

FIGURE 5 | Quantitative PCR analysis of selected genes encoding matrix-associated proteins in different biofilm phases. The relative expression was calculated based on the $\Delta \mathrm{Ct}$ values. The $16 \mathrm{~S}$ rRNA gene was used as a reference. Average values from three experimental replicates are shown. Correlation between the abundance of proteins and their corresponding gene transcripts is shown in Supplementary Figure 1. 


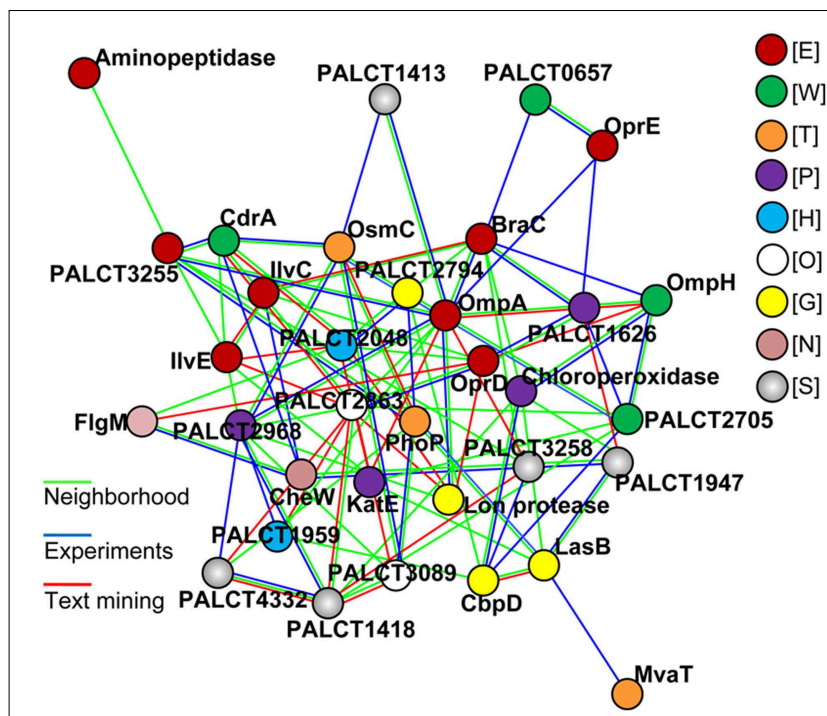

FIGURE 6 | The most significant protein-protein interaction model for proteins with a changed abundance in the matrix during biofilm development. Lines in color indicate different interactions defined by the STRING. Proteins in color indicate different COG categories: E, amino acid transport and metabolism; W, extracellular structures; T, Signal transduction; P, inorganic ion transport and metabolism; $\mathrm{H}$, coenzyme transport and metabolism; O, post-translational modifications, protein turnover, chaperones; G, Carbohydrate transport and metabolism; N, cell motility; S, function unknown.

\section{Matrix-associated Proteins and Nutrient Metabolism}

The polysaccharides of the biofilm matrix act as an external digestive system by maintaining a close proximity between extracellular enzymes and bacterial cells, enabling them to metabolize dissolved, colloidal and solid organic carbons (HallStoodley et al., 2004). Jiao et al. (2011) showed that natural biofilms contain CAZymes, which are likely to be involved in matrix degradation. One example is cellulase, which participates in the recycling of extracellular polysaccharides for nutrients or in biofilm dissolution. In the present study, we found that several proteins that accumulated in the matrix during biofilm development were related to nutrient metabolism, such as amino acid aminotransferase and aminopeptidase (Figure 3A). The increase in these proteins could be explained by biofilm growth-related stresses such as nutrient limitation. In addition to proteins related to amino acid metabolism, outer membrane proteins including transporters and porins also showed an increased abundance in the matrix from earlier to later phases. Outer membrane proteins are frequently identified in the extracellular space. The presence of these proteins in the matrix suggests that they may have roles in the biofilm other than nutrient transport. For example, it has been reported that OmpA is overexpressed in Escherichia coli during biofilm formation and that large quantities of OmpA can be secreted into the extracellular space (Gophna et al., 2004; Orme et al., 2006). OmpA is essential for biofilm development and resistance to antimicrobial agents in Sodalis glossinidius (Maltz et al., 2012). The ompA mutant strain fails to form biofilms in vitro and is unable to colonize the tsetse gut unless endogenous symbiotic bacteria are present (Maltz et al., 2012). In the present study, we consistently found that mutation of the ompA gene in $P$. aeruginosa ATCC27853 reduced the substrate coverage, thickness and concentration of exopolysaccharides. Collectively, the increase in nutrient metabolism-related and outer membrane proteins in the matrix may be a strategy for overcoming nutrient limitation and maintaining the stability of the biofilm. The reduced amounts of exopolysaccharides in the $\triangle o m p A$ biofilm also suggested a complex correlation between proteins and polysaccharides in the matrix.

\section{Matrix-associated Proteins and Stress Resistance}

Biofilm development is coupled to enhanced stress resistance. Polysaccharides, as the best studied components of the matrix, have been reported to provide protection against a variety of environmental stresses, including antibiotics, $\mathrm{pH}$ shifts, osmotic shock and desiccation (Hall-Stoodley et al., 2004; Flemming and Wingender, 2010). Here, we found that increased stress resistance during biofilm development also could be explained by the enhanced abundance of particular proteins in the matrix (Figure 3B). Catalase and chloroperoxidase, which act directly on peroxides, showed an increased abundance in the matrix. Based on previous studies (Stewart et al., 2000), catalases protect aggregated bacteria by preventing the complete penetration of hydrogen peroxide into the biofilm. CatE was identified as a component of the biofilm matrix formed by $P$. aeruginosa PAO1 (Toyofuku et al., 2012), and the abundance of catalase genes were found to be enriched in later compared to earlier phases of biofilm development in our previous study examining the intertidal biofilm community (Zhang et al., 2013a). Moreover, methionine sulfoxide reductase also increased during biofilm development. Methionine sulfoxide reductase has been demonstrated to play an important role in oxidative stress resistance (Lei et al., 2011), and it also reduces oxidized methionine in surface adhesion, thus facilitating biofilm formation under oxidative stress (Mintz et al., 2002). In addition to the oxidative stress-related proteins, proteins contributing to heat shock stress resistance were also increased in the matrix during biofilm development. Heat shock proteins have been reported to promote biofilm formation in several species, and they are up-regulated in biofilms compared to the planktonic state (Ito et al., 2009; Kuczyńska-Wiśnik et al., 2010). The actual function of heat shock proteins in the matrix could be to protect and stabilize proteins as chaperones; however, extracellular heat shock proteins are involved in the modulation of host immunity responses (Retzlaff et al., 1994). In a previous study on biofilms from acid mine drainage (Jiao et al., 2011), cold shock rather than heat shock proteins were identified in the biofilm matrix, suggesting that biofilms from different environments may take different strategies for stress resistance. All of these results suggested a correlation between increased stress resistance during 
TABLE 1 | CLSM-based characteristics of $P$. aeruginosa ATCC27853 and mutant biofilm structure in the mature II phase (phase IV, $96 \mathrm{~h}$ ).

\begin{tabular}{lcccc}
\hline Strains & Substrate coverage $(\%)$ & Average thickness $(\boldsymbol{\mu} \mathbf{m})$ & Maximum thickness $(\boldsymbol{\mu} \mathbf{m})$ & Exopolysaccharide $(\boldsymbol{\mu g} / \mathbf{1 0 \wedge 9 C F U ) ~}$ \\
\hline Wild type & $72.34 \pm 2.12$ & $6.98 \pm 0.71$ & $37.56 \pm 3.54$ & $32.45 \pm 1.45$ \\
$\Delta o p r D$ & $65.36 \pm 2.83$ & $5.89 \pm 0.50$ & $24.12 \pm 1.41^{\star}$ & $30.47 \pm 7.16$ \\
$\Delta O m p A$ & $34.79 \pm 7.07^{\star}$ & $3.28 \pm 0.35^{\star}$ & $19.54 \pm 3.53^{\star}$ & $14.67 \pm 2.14^{\star}$ \\
$\Delta c b p D$ & $45.87 \pm 1.41^{\star}$ & $4.31 \pm 0.71^{\star}$ & $14.56 \pm 0.72^{\star}$ & $12.39 \pm 0.85^{\star}$ \\
\hline
\end{tabular}

"*” Indicates a significant change compared with the wild type strain.

biofilm development and the dynamics of matrix-associated proteins.

\section{Secreted Proteins in the Biofilm Matrix}

Secreted proteins are important parts of the biofilm matrix, and in the present study, several secreted proteins identified changed their abundance during biofilm development. Thus, the potential functions of secreted proteins in the matrix are discussed. One example is the elastase LasB, which is an important virulence factor and permits escape from phagocytosis (Kuang et al., 2011). Moreover, LasB can also influence the formation and architecture of mucoid $P$. aeruginosa SG81 biofilm as a result of changes in the matrix composition and properties (Tielen et al., 2010). Another secreted protein with a changed abundance in the matrix was $\mathrm{CbpD}$, a chitinbinding protein. Chitin-binding proteins can enhance the rate of bacterial infection in human intestinal cells (Chaudhuri et al., 2010); chitin binding proteins also are involved in adaptation to environmental nutrient gradients, tolerance to stress and protection against predators (Pruzzo et al., 2008). Considering all of these findings, we hypothesize that the increased abundance of the two secreted proteins in the biofilm matrix may contribute to both pathogenesis and biofilm stability. Moreover, three secreted proteins in the matrix are likely to be novel effectors of the TTSS in the P. aeruginosa ATCC27853 biofilm and their abundance in the matrix decreased during biofilm development (Figure 4 and Supplementary Table 4). Effectors of the secretion system are powerful weapons used by bacteria to infect hosts (Hauck et al., 2003; Geddes et al., 2007). The increase in LasB and $\mathrm{CbpD}$ and decrease in putative effectors suggest that different virulence strategies are adopted by biofilms at different phases.

Many proteins with modulated abundance in the matrix were cytoplasmic proteins. This result was consistent with findings in a previous study (Jiao et al., 2011) that examined biofilm matrixassociated proteins. In addition, increasing evidence shows that many previously-known intracellular proteins such as catalase and heat shock proteins also exert functions outside of the cell. In a recent study (Foulston et al., 2014), Foulston et al. reported that the extracellular matrix of the Staphylococcus aureus biofilm comprises cytoplasmic proteins that associate with the cell surface in response to decreasing $\mathrm{pH}$. While the presence of these proteins could be due to unknown secretion processes, another explanation is programmed cell death in the biofilm community, which naturally causes lysis (Bayle, 2007). The results represented in the present study suggested close interactions among the changed proteins; and mutation of the selected proteins in the center of the interaction network attenuated biofilm formation. In addition, based on expression profiling, the dynamics of matrix-associated proteins seem to be regulated by the bacteria. Furthermore, the functions and phenotype shifts observed during biofilm development were consistent with the dynamic patterns of the matrixassociated proteins. For example, as mentioned above, the enhanced stress resistance, nutrient acquisition and pathogenesis during biofilm development could be explained by the dynamic pattern of matrix-associated proteins. Taken together, we propose that the proteins in the matrix would be well organized and act as an integral part of the biofilm, whereas most of the proteins, including extracellular, outer membrane and cytoplasmic proteins, are functional components of the biofilm. The present study has elucidated potential roles of extracellular matrix-associated proteins in P. aeruginosa ATCC27853 biofilm development from a dynamic perspective, aiming to provide resources for the mechanistic understanding and control of bacterial biofilms. As an extension, our future focus will include interactions of particular proteins identified in the present work and their roles in biofilm development. For example, three secreted proteins in the matrix are likely to be novel effectors of the TTSS in the P. aeruginosa, and their functions await further characterization.

\section{Acknowledgments}

This study was supported by awards from the Sanya Institute of Deep Sea Science and Engineering, the Chinese Academy of Sciences (SIDSSE, CAS) (SIDSSE-201206), COMRA program of China (COMRRDA12SC01), and General Research Fund (661611) from HKSAR government to PQ.

\section{Supplementary Material}

The Supplementary Material for this article can be found online at: http://journal.frontiersin.org/article/10.3389/fcimb. 2015.00040/abstract 


\section{References}

Arciola, C. R., Campoccia, D., Gamberini, S., Donati, M. E., Pirini, V., Visai, L., et al. (2005). Antibiotic resistance in exopolysaccharide-forming Staphylococcus epidermidis clinical isolates from orthopaedic implant infections. Biomaterials 26, 6530-6535. doi: 10.1016/j.biomaterials.2005.04.031

Bayle, K. W. (2007). The biological role of death and lysis in biofilm development. Nat. Rev. Microbiol. 5, 721-726. doi: 10.1038/nrmicro1743

Borlee, B. R., Goldman, A. D., Murakami, K., Samudrala, R., Wozniak, D. J., and Parsek, M. R. (2010). Pseudomonas aeruginosa uses a cyclic-di-GMPregulated adhesin to reinforce the biofilm extracellular matrix. Mol. Microbiol. 75, 827-842. doi: 10.1111/j.1365-2958.2009.06991.x

Cantarel, B. L., Coutinho, P. M., Rancurel, C., Bernard, T., Lombard, V., and Bernard, H. B. (2009). The Carbohydrate-Active EnZymes database (CAZy): an expert resource for Glycogenomics. Nucleic Acids Res. 37, 233-238. doi: 10.1093/nar/gkn663

Chaudhuri, S., Bruno, J. C., Alonzo, F., Xayarath, B., Cianciotto, N. P., and Freitag, N. E. (2010). Contribution of chitinases to Listeria monocytogenes pathogenesis. Appl. Environ. Microbiol. 76, 7302-7305. doi: 10.1128/AEM.01338-10

Colvin, K. M., Gordon, V. D., Murakami, K., Borlee, B. R., Wozniak, D. J., Wong, G. C., et al. (2011). The Pel polysaccharide can serve a structural and protective role in the biofilm matrix of Pseudomonas aeruginosa. PLoS Pathog. 7:1001264. doi: 10.1371/journal.ppat.1001264

Delcher, A. L., Bratke, K. A., Powers, E. C., and Salzberg, S. L. (2007). Identifying bacterial genes and endosymbiont DNA with Glimmer. Bioinformatics 23, 673-679. doi: 10.1093/bioinformatics/btm009

Fang, X., Fang, Z., Zhao, J., Zou, Y., Li, T.,Wang, J., et al. (2012). Draft genome sequence of Pseudomonas aeruginosa strain ATCC27853. J. Bacteriol. 194, 3755-3755. doi: 10.1128/JB.00690-12

Fass, R. J., and Barnishan, J. (1979). Minimal inhibitory concentrations of 34 antimicrobial agents for control strains Escherichia coli ATCC 25922 and Pseudomonas aeruginosa ATCC 27853. Antimicrob. Agents Chemother. 16, 622-624. doi: 10.1128/AAC.16.5.622

Flemming, H. C., and Wingender, J. (2010). The biofilm matrix. Nat. Rev. Microbiol. 8, 623-633. doi: 10.1038/nrmicro2415

Foulston, L., Elsholz, A. K. W., DeFrancesco, A. S., and Losick, R. (2014). The extracellular matrix of Staphylococcus aureus biofilms comprises cytoplasmic proteins that associate with the cell surface in response to decreasing $\mathrm{pH}$. MBio 5:e1667-14. doi: 10.1128/mBio.01667-14

Gallaher, T. K., Wu, S., Webster, P., and Aguilera, R. (2006). Identification of biofilm proteins in non-typeable Haemophilus influenzae. BMC Microbiol. 6:65. doi: 10.1186/1471-2180-6-65

Geddes, K., Cruz, III. F., and Heffron, F. (2007). Analysis of cells targeted by Salmonella type III secretion in vivo. PLoS Pathog. 3:e196. doi: 10.1371/journal.ppat.0030196

Giraud, C., Bernard, C., Ruer, S., and De Bentzmann, S. (2010). Biological 'glue'and 'Velcro': molecular tools for adhesion and biofilm formation in the hairy and gluey bug Pseudomonas aeruginosa. Environ. Microbiol. Rep. 2, 343-358. doi: 10.1111/j.1758-2229.2009.00070.x

Goller, C. C., and Seed, P. C. (2010). Revisiting the Escherichia coli polysaccharide capsule as a virulence factor during urinary tract infection: contribution to intracellular biofilm development. Virulence 1, 333-337. doi: 10.4161/viru.1.4.12388

Gophna, U., Ideses, D., Rosen, R., Grundland, A., and Ron, E. Z. (2004). OmpA of a septicemic Escherichia coli O78-secretion and convergent evolution. Int. J. Med. Microbiol. 29, 373-381. doi: 10.1016/j.ijmm.2004.08.004

Guo, S., Alshamy, I., Hughes, K. T., and Chevance, F. F. (2014). Analysis of factors that affect FlgM-dependent type III secretion for protein purification with Salmonella enterica serovar typhimurium. J. Bacteriol. 196, 2333-2347. doi: 10.1128/JB.01572-14

Hall-Stoodley, L., Costerton, J. W., and Stoodley. P. (2004). Bacterial biofilms: from the natural environment to infectious diseases. Nat. Rev. Microbiol. 2, 95-108. doi: $10.1038 /$ nrmicro821

Han, Z., Sunm, J., Zhang, Y., He, F., Xu, Y., Matsumura, K., et al. (2013). iTRAQbased proteomic profiling of the barnacle Balanus amphitrite in response to the antifouling compound meleagrin. J. Proteome Res. 12, 2090-2100. doi: $10.1021 /$ pr301083e
Hauck, P., Thilmony, R., and He, S. Y. (2003). A Pseudomonas syringae type III effector suppresses cell wall-based extracellular defense in susceptible Arabidopsis plants. Proc. Natl. Acad. Sci. U.S.A. 100, 8577-8582. doi: $10.1073 /$ pnas. 1431173100

Hewson, W. D., and Hage, L. P. (2012). Peroxidases, catalases, and chloroperoxidase. Porphyrins 7, 295-332.

Ito, A., Taniuchi, A., May, T., Kawata, K., and Okabe, S. (2009). Increased antibiotic resistance of Escherichia coli in mature biofilms. Appl. Environ. Microbiol. 75, 4093-4100. doi: 10.1128/AEM.02949-08

Jehl, M. A., Arnold, R., and Rattei, T. (2001). Effective-a database of predicted secreted bacterial proteins. Nucleic Acids Res. 39, D591-D595. doi: $10.1093 / \mathrm{nar} / \mathrm{gkq} 1154$

Jiao, Y., D’Haeseleer, P., Dill, B. D, Shah, M., VerBerkmoes, N. C., and Hettich, R. L. (2011). Identification of biofilm matrix-associated proteins from an acid mine drainage microbial community. Appl. Environ. Microbiol. 77, 5230-5237. doi: 10.1128/AEM.03005-10

Kanehisa, M., and Goto, S. (2000). KEGG: kyoto encyclopedia of genes and genomes. Nucleic Acids Res. 28, 27-30. doi: 10.1093/nar/28.1.27

Kuang, Z., Hao, Y.,Walling, B. E., Jeffries, J. L., Ohman, D. E., and Lau, G. W. (2011). Pseudomonas aeruginosa elastase provides an escape from phagocytosis by degrading the pulmonary surfactant protein-A. PLoS ONE 6:e27091. doi: 10.1371/journal.pone.0027091

Kuczyńska-Wiśnik, D., Matuszewska, E., and Laskowska, E. (2010). Escherichia coli heat-shock proteins IbpA and IbpB affect biofilm formation by influencing the level of extracellular indole. Microbiology 156, 148-157. doi: 10.1099/mic.0.032334-0

Lei, Y., Zhang, Y., Guenther, B. D., Kreth, J., and Herzberg, M. C. (2011). Mechanism of adhesion maintenance by methionine sulphoxide reductase in Streptococcus gordonii. Mol. Microbiol. 80, 726-738. doi: 10.1111/j.13652958.2011.07603.x

Lewenza, S. (2013). Extracellular DNA-induced antimicrobial peptide resistance mechanisms in Pseudomonas aeruginosa. Front. Microbiol. 4:21. doi: 10.3389/fmicb.2013.00021

Lilic, M., Vujanac, M., and Stebbins, C. A. (2006). Common structural motif in the binding of virulence factors to bacterial secretion chaperones. Mol. Cell 21, 653-664. doi: 10.1016/j.molcel.2006.01.026

Liu, H., and Fang, H. H. (2002). Extraction of extracellular polymeric substances (EPS) of sludges. J. Biotechnol. 95, 249-256. doi: 10.1016/S0168-1656(02) 00025-1

Mah, T.-F. C., and O'Toole, G. A. (2001). Mechanisms of biofilm resistance to antimicrobial agents. Trends Microbiol. 9, 34-39. doi: 10.1016/S0966842X(00)01913-2

Maltz, M. A., Weiss, B. L., O’Neill, M., Wu, Y., and Aksoy, S. (2012). OmpAmediated biofilm formation is essential for the commensal bacterium Sodalis glossinidius to colonize the tsetse fly gut. Appl. Environ. Microbiol. 78, 7760-7768. doi: 10.1128/AEM.01858-12

Mintz, K. P., Moskovitz, J., Wu, H., and Fives-Taylor, P. M. (2002). Peptide methionine sulfoxide reductase (MsrA) is not a major virulence determinant for the oral pathogen Actinobacillus actinomycetemcomitans. Microbiology 148, 3695-3703.

Myszka, K., and Czaczyk, K. (2009). Characterization of adhesive exopolysaccharide (EPS) produced by Pseudomonas aeruginosa under starvation conditions. Curr. Microbiol. 58, 541-546. doi: 10.1007/s00284-009-9365-3

Orme, R., Douglas, C. W., Rimmer, S., and Webb, M. (2006). Proteomic analysis of Escherichia coli biofilms reveals the overexpression of the outer membrane protein OmpA. Proteomics 6, 4269-4277. doi: 10.1002/pmic.200600193

O’Toole, G., Kaplan, H. B., and Kolter, R. (2000). Biofilm formation as microbial development. Annu. Rev. Microbiol. 54, 49-79. doi: 10.1146/annurev.micro.54.1.49

Pruzzo, C., Vezzulli, L., and Colwell, R. R. (2008). Global impact of Vibrio cholerae interactions with chitin. Environ. Microbiol. 10, 1400-1410. doi: 10.1111/j.14622920.2007.01559.x

Retzlaff, C., Yamamoto, Y., Hoffman, P. S., Friedman, H., and Klein, T. W. (1994). Bacterial heat shock proteins directly induce cytokine mRNA and interleukin-1 secretion in macrophage cultures. Infect. Immun. 62, 5689-5693.

Rinaudi, L., Fujishige, N. A., Hirsch, A. M., Banchioa, E., Zorreguietad, A., and Giordanoa, W. (2006). Effects of nutritional and environmental conditions on 
Sinorhizobium meliloti biofilm formation. Res. Microbiol. 157, 867-875. doi: 10.1016/j.resmic.2006.06.002

Sansom, F. M., Tang, L., Ralton, J. E., Saunders, E. C., Naderer, T., and McConvill, M. J. (2013). Leishmania major methionine sulfoxide reductase A is required for resistance to oxidative stress and efficient replication in macrophages. PLoS ONE 8:e56064. doi: 10.1371/journal.pone.0056064

Speziale, P., Pietrocola, G., Foster, T. J., and Geoghegan, J. A. (2014). Proteinbased biofilm matrices in Staphylococci. Front. Cell. Infect. Microbiol. 4:171. doi: 10.3389/fcimb.2014.00171

Sriramulu, D. D., Lünsdorf, H., Lam, J. S., and Römling, U. (2005). Microcolony formation: a novel biofilm model of Pseudomonas aeruginosa for the cystic fibrosis lung. J. Med. Microbiol. 54, 667-676. doi: 10.1099/jmm.0.45969-0

Stewart, P. S., Peyton, B., Drury, W. J., and Murga, R. (1993). Quantitative observations of heterogeneities in Pseudomonas aeruginosa biofilms. Appl. Environ. Microbiol. 59, 327-329.

Stewart, P. S., Roe, F., Rayner, J. G., Elkins, Z., Lewandowski, U. A., Ochsner, U. A., et al. (2000). Effect of catalase on hydrogen peroxide penetration into Pseudomonas aeruginosa biofilms. Appl. Environ. Microbiol. 66, 8. doi: 10.1128/AEM.66.2.836-838.2000

Tatusov, R. L., Natale, D. A, Garkavtsev, I. V., Tatusova, T. A., Shankavaram, U. T., Rao, B. S., et al. (2001). The COG database: new developments in phylogenetic classification of proteins from complete genomes. Nucleic Acids Res. 29, 22-28. doi: 10.1093/nar/29.1.22

Tielen, P., Rosenau, F., Wilhelm, S., Jaeger, K. E., Flemming, H. C., and Wingender, J. (2010). Extracellular enzymes affect biofilm formation of mucoid Pseudomonas aeruginosa. Microbiology 156, 2239-2252. doi: 10.1099/mic.0.037036-0

Toyofuku, M., Roschitzki, B., Riedel, K., and Eberl, L. (2012). Identification of proteins associated with the Pseudomonas aeruginosa biofilm extracellular matrix. J. Proteome Res. 11, 4906-4915. doi: 10.1021/pr300395j

Von Mering, C., Huynen, M., Jaeggi, D., Schmidt, S., Bork, P., and Snel, B. (2003). STRING: a database of predicted functional associations between proteins. Nucleic Acids Res. 31, 258-261. doi: 10.1093/nar/gkg034
Waite, R. D., Papakonstantinopoulou, A., Littler, E., and Curtis, M. A. (2005). Transcriptome analysis of Pseudomonas aeruginosa growth: comparison of gene expression in planktonic cultures and developing and mature biofilms. J. Bacteriol. 187, 6571-6576. doi: 10.1128/JB.187.18.6571-65 76.2005

Wiese, S., Reidegeld, K. A., Meyer, H. E., and Warscheid, B. (2007). Protein labeling by iTRAQ: a new tool for quantitative mass spectrometry in proteome research. Proteomics 7, 340-350. doi: 10.1002/pmic.200600422

$\mathrm{Xu}, \mathrm{D}$., and Zhang, Y. (2012). Ab initio protein structure assembly using continuous structure fragments and optimized knowledge-based force field. Proteins 80, 1715-1735. doi: 10.1002/prot.24065

Zhang, W. P., Wang, Y., Lee, O. O., Tian, R. M., Cao, H. L., Gao, Z. M., et al. (2013a). Adaptation of intertidal biofilm communities is driven by metal ion and oxidative stresses. Sci. Rep. 3:3180. doi: 10.1038/srep03180

Zhang, W. P., Wang, Y., Song, Y. H., Wang, T. T., Xu, S. J., Peng, Z., et al. (2013b). A type VI secretion system regulated by OmpR in Yersinia pseudotuberculosis functions to maintain intracellular $\mathrm{pH}$ homeostasis. Environ. Microbiol. 15, 557-569. doi: 10.1111/1462-2920.12005

Zhang, X., Bishop, P. L., and Kinkle, B. K. (1999). Comparison of extraction methods for quantifying extracellular polymers in biofilms. Water Sci. Technol. 39, 211-218. doi: 10.1016/S0273-1223(99)00170-5

Conflict of Interest Statement: The authors declare that the research was conducted in the absence of any commercial or financial relationships that could be construed as a potential conflict of interest.

Copyright (C) 2015 Zhang, Sun, Ding, Lin, Tian, Lu, Liu, Shen and Qian. This is an open-access article distributed under the terms of the Creative Commons Attribution License (CC BY). The use, distribution or reproduction in other forums is permitted, provided the original author(s) or licensor are credited and that the original publication in this journal is cited, in accordance with accepted academic practice. No use, distribution or reproduction is permitted which does not comply with these terms. 